Nonlinear Mathematical Physics 1997, V.4, N 1-2, 124-128.

\title{
Continuity Equation in Nonlinear Quantum Mechanics and the Galilei Relativity Principle
}

Wilhelm FUSHCHYCH and Vyacheslav BOYKO

Institute of Mathematics of the National Ukrainian Academy of Sciences,

3 Tereshchenkivs'ka Street, 252004 Kyïv, Ukraïna

Abstract

Classes of the nonlinear Schrödinger-type equations compatible with the Galilei relativity principle are described. Solutions of these equations satisfy the continuity equation.

The continuity equation is one of the most fundamental equations of quantum mechanics

$$
\frac{\partial \rho}{\partial t}+\vec{\nabla} \cdot \vec{j}=0
$$

Depending on definition of $\rho$ (density) and $\vec{j}=\left(j^{1}, \ldots, j^{n}\right)$ (current), we can construct essentially different quantum mechanics with different equations of motion, which are distinct from classical linear Schrödinger, Klein-Gordon-Fock, and Dirac equations.

In this paper we describe wide classes of the nonlinear Schrödinger-type equations compatible with the Galilei relativity principle and their solutions satisfy the continuity equation.

1. At the beginning we study a symmetry of the continuity equation considering $(\rho, \vec{j})$ as dependent variables related by (1).

Theorem 1 The invariance algebra of equation (1) is an infinite-dimensional algebra with basis operators

$$
X=\xi^{\mu}(x) \frac{\partial}{\partial x_{\mu}}+\left(a^{\mu \nu}(x) j^{\nu}+b^{\mu}(x)\right) \frac{\partial}{\partial j^{\mu}},
$$

where $j^{0} \equiv \rho ; \xi^{\mu}(x)$ are arbitrary smooth functions; $x=\left(x_{0}=t, x_{1}, x_{2}, \ldots, x_{n}\right) \in$ $\mathbf{R}^{n+1} ; a^{\mu \nu}(x)=\frac{\partial \xi^{\mu}}{\partial x_{\nu}}-\delta_{\mu \nu}\left(\frac{\partial \xi^{i}}{\partial x_{i}}+C\right) ; C=$ const, $\delta_{\mu \nu}$ is the Kronecker delta; $\mu, \nu, i=$ $0,1, \ldots, n,\left(b^{0}(x), b^{1}(x), \ldots, b^{n}(x)\right)$ is an arbitrary solution of equation (1).

Here and below we imply summation over repeated indices.

Corollary 1 The generalized Galilei algebra [1]

$$
A G_{2}(1, n)=<P_{\mu}, J_{a b}, G_{a}, D^{(1)}, A>
$$

is a subalgebra of algebra (2).

Corollary 2 The conformal algebra [1]

$$
A P_{2}(1, n)=A C(1, n)=<P_{\mu}, J_{a b}, J_{0 a}, D^{(2)}, K_{\mu}>
$$

is a subalgebra of algebra (2). 
We use the following designations in (3) and (4)

$$
\begin{aligned}
& P_{\mu}=\partial_{\mu}, \quad J_{a b}=x_{a} \partial_{b}-x_{b} \partial_{a}+j^{a} \partial_{j^{b}}-j^{b} \partial_{j^{a}}, \quad(a<b) \\
& G_{a}=x_{0} \partial_{a}+\rho \partial_{j^{a}}, \quad J_{0 a}=x_{a} \partial_{0}+x_{0} \partial_{a}+j^{a} \partial_{\rho}+\rho \partial_{j^{a}}, \\
& D^{(1)}=2 x_{0} \partial_{0}+x_{a} \partial_{a}-n \rho \partial_{\rho}-(n+1) j^{a} \partial_{j^{a}}, \quad D^{(2)}=x_{\mu} \partial_{\mu}-n \rho \partial_{\rho}-n j^{a} \partial_{j^{a}}, \\
& A=x_{0}^{2} \partial_{0}+x_{0} x_{a} \partial_{a}-n x_{0} \rho \partial_{\rho}+\left(x_{a} \rho-(n+1) x_{0} j^{a}\right) \partial_{j^{a}}, \\
& K_{\mu}=2 x_{\mu} D^{(2)}-x_{\nu} x^{\nu} g_{\mu i} \partial_{i}-2 x^{\nu} S_{\mu \nu}, \quad S_{\mu \nu}=g_{\mu i} j^{\nu} \partial_{j^{i}}-g_{\nu i} j^{\mu} \partial_{j^{i}}, \\
& g_{\mu \nu}=\left\{\begin{array}{rl}
1, & \mu=\nu=0 \\
-1, & \mu=\nu \neq 0 \\
0 . & \mu \neq \nu,
\end{array} \quad \mu, \nu, i=0,1 \ldots, n ; a, b=1,2, \ldots, n .\right.
\end{aligned}
$$

Corollary 3 The continuity equation satisfies the Galilei relativity principle as well as the Lorentz-Poincare-Einstein relativity principle.

Thus, depending on the definition of $\rho$ and $\vec{j}$, we come to different quantum mechanics.

2. Let us consider the scalar complex-valued wave functions and define $\rho$ and $\vec{j}$ in the following way

$$
\rho=f\left(u u^{*}\right), \quad j^{k}=-\frac{1}{2} i g\left(u u^{*}\right)\left(\frac{\partial u}{\partial x_{k}} u^{*}-u \frac{\partial u^{*}}{\partial x_{k}}\right)+\frac{\partial \varphi\left(u u^{*}\right)}{\partial x_{k}}, \quad k=1,2, \ldots, n .
$$

where $f, g, \varphi$ are arbitrary smooth functions, $f \neq$ const, $g \neq 0$. Without loss of generality, we assume that $f \equiv u u^{*}$.

Let us describe all functions $g\left(u u^{*}\right), \varphi\left(u u^{*}\right)$ for continuity equation (1), (5) to be compatible with the Galilei relativity principle, defined by the following transformations:

$$
t \rightarrow t^{\prime}=t, \quad x_{a} \rightarrow x_{a}^{\prime}=x_{a}+v_{a} t .
$$

Here we do not fix transformation rules for the wave function $u$.

Theorem 2 If $\rho$ and $\vec{j}$ are defined according to formula (5), then the continuity equation (1) is Galilei-invariant iff

$$
\rho=u u^{*}, \quad j^{k}=-\frac{1}{2} i\left(\frac{\partial u}{\partial x_{k}} u^{*}-u \frac{\partial u^{*}}{\partial x_{k}}\right)+\frac{\partial \varphi\left(u u^{*}\right)}{\partial x_{k}}, \quad k=1,2, \ldots, n .
$$

The corresponding generators of Galilei transformations have the form

$$
G_{a}=x_{0} \partial_{a}+i x_{a}\left(u \partial_{u}-u^{*} \partial_{u^{*}}\right), \quad a=1,2, \ldots, n .
$$

If in (6)

$$
\varphi=\lambda u u^{*}, \quad \lambda=\text { const },
$$

then the continuity equation (1), (6), (7) coincides with the Fokker-Planck equation

$$
\frac{\partial \rho}{\partial t}+\vec{\nabla} \cdot \vec{j}+\lambda \Delta \rho=0
$$


where

$$
\rho=u u^{*}, \quad j^{k}=-\frac{1}{2} i\left(\frac{\partial u}{\partial x_{k}} u^{*}-u \frac{\partial u^{*}}{\partial x_{k}}\right), \quad k=1,2, \ldots, n .
$$

The continuity equation (1), (6), (7) was considered in $[2,6]$.

Let us invistigate the symmetry of the nonlinear Schrödinger equation

$$
i u_{0}+\frac{1}{2} \Delta u+i \frac{\Delta \varphi\left(u u^{*}\right)}{2 u u^{*}} u=F\left(u u^{*},\left(\vec{\nabla}\left(u u^{*}\right)\right)^{2}, \Delta\left(u u^{*}\right)\right) u,
$$

where $F$ is an arbitrary real smooth function.

For the solutions of equation (10), equation (1), (6) is satisfied and is compatible with the Galilei relativity principle. Schrödinger equations in the form of (10), when $\varphi\left(u u^{*}\right)=\lambda u u^{*}$ for fixed function $F$, were considered in [1]-[8].

In terms of the phase and amplitude $(u=R \exp (i \Theta))$, equation (10) has the form

$$
\begin{aligned}
& R_{0}+R_{k} \Theta_{k}+\frac{1}{2} R \Delta \Theta+\frac{1}{2 R} \Delta \varphi=0, \\
& \Theta_{0}+\frac{1}{2} \Theta_{k}^{2}-\frac{1}{2 R} \Delta R+F\left(R^{2},\left(\vec{\nabla}\left(R^{2}\right)\right)^{2}, \Delta R^{2}\right)=0 .
\end{aligned}
$$

Theorem 3 The maximal invariance algebras for system (11), if $F=0$, are the following:

$$
\text { 1. }<P_{\mu}, J_{a b}, Q, G_{a}, D>
$$

when $\varphi$ is an arbitrary function;

2. $<P_{\mu}, J_{a b}, Q, G_{a}, D, I, A>$

when $\varphi=\lambda R^{2}, \lambda=$ const.

In (12) and (13) we use the following designations:

$$
\begin{aligned}
& P_{\mu}=\partial_{\mu}, \quad J_{a b}=x_{a} \partial_{x_{b}}-x_{b} \partial_{x_{a}}, \quad a<b \\
& G_{a}=x_{0} \partial_{x_{a}}+i x_{a} \partial_{\Theta}, \quad Q=\partial_{\Theta}, \quad D=2 x_{0} \partial_{x_{0}}+x_{a} \partial_{x_{a}}, \quad I=R \partial_{R}, \\
& A=x_{0}^{2} \partial_{x_{0}}+x_{0} x_{a} \partial_{x_{a}}-\frac{n}{2} x_{0} R \partial_{R}+\frac{1}{2} x_{a}^{2} \partial_{\Theta}, \\
& \mu=0,1, \ldots, n ; \quad a, b=1,2, \ldots, n .
\end{aligned}
$$

Algebra (13) coincides with the invariance algebra of the linear Schrödinger equation.

Corollary 4 System (11), (7) is invariant with respect to algebra (13) if

$$
F=R^{-1} \Delta R N\left(\frac{R \Delta R}{(\vec{\nabla} R)^{2}}\right),
$$

where $N$ is an arbitrary real smooth function. 
3. Let us consider a more general system than (10)

$$
i u_{0}+\frac{1}{2} \Delta u=\left(F_{1}+i F_{2}\right) u,
$$

where $F_{1}, F_{2}$ are arbitrary real smooth functions,

$$
F_{m}=F_{m}\left(u u^{*},\left(\vec{\nabla}\left(u u^{*}\right)\right)^{2}, \Delta\left(u u^{*}\right)\right) u, \quad m=1,2 .
$$

The structure of functions $F_{1}, F_{2}$ may be described in form (16) by virtue of conditions for system (15) to be Galilei-invariant.

In terms of the phase and amplitude, equation (15) has the form

$$
R_{0}+R_{k} \Theta_{k}+\frac{1}{2} R \Delta \Theta-R F_{2}=0, \quad \Theta_{0}+\frac{1}{2} \Theta_{k}^{2}-\frac{1}{2 R} \Delta R+F_{1}=0
$$

where $F_{m}=F_{m}\left(R^{2},\left(\vec{\nabla}\left(R^{2}\right)\right)^{2}, \Delta R^{2}\right), \quad m=1,2$.

Theorem 4 System (17) is invariant with respect to the generalized Galilei algebra $A G_{2}(1, n)=<P_{\mu}, J_{a b}, G_{a}, Q, \widetilde{D}, A>$ if it has the form

$$
\begin{aligned}
& R_{0}+R_{k} \Theta_{k}+\frac{1}{2} R \Delta \Theta-R^{1+4 / n} M\left(\frac{(\vec{\nabla} R)^{2}}{R^{2+4 / n}} ; \frac{\Delta R}{R^{1+4 / n}}\right)=0, \\
& \Theta_{0}+\frac{1}{2} \Theta_{k}^{2}-\frac{1}{2 R} \Delta R+R^{4 / n} N\left(\frac{(\vec{\nabla} R)^{2}}{R^{2+4 / n}} ; \frac{\Delta R}{R^{1+4 / n}}\right)=0,
\end{aligned}
$$

where $N, M$ are arbitrary real smooth functions. The basis operators of the algebra $A G_{2}(1, n)$ are defined by (14) and $\widetilde{D}=D-\frac{n}{2} I$.

Theorem 5 System (17) is invariant with respect to algebra (13) if it has the form

$$
\begin{aligned}
& R_{0}+R_{k} \Theta_{k}+\frac{1}{2} R \Delta \Theta-\Delta R M\left(\frac{R \Delta R}{(\vec{\nabla} R)^{2}}\right)=0, \\
& \Theta_{0}+\frac{1}{2} \Theta_{k}^{2}-\frac{1}{2 R} \Delta R+\frac{\Delta R}{R} N\left(\frac{R \Delta R}{(\vec{\nabla} R)^{2}}\right)=0,
\end{aligned}
$$

where $N, M$ are arbitrary real smooth functions.

System (18) written in terms of the wave function has the form

$$
i u_{0}+\frac{1}{2} \Delta u=\frac{\Delta|u|}{|u|}\left(N\left(\frac{|u| \Delta|u|}{(\vec{\nabla}|u|)^{2}}\right)+i M\left(\frac{|u| \Delta|u|}{(\vec{\nabla}|u|)^{2}}\right)\right) u .
$$

Equation (19) is equivalent to the following equation

$$
i u_{0}+\frac{1}{2} \Delta u=\frac{\Delta\left(u u^{*}\right)}{\left(u u^{*}\right)}\left(\tilde{N}\left(\frac{\left(u u^{*}\right) \Delta\left(u u^{*}\right)}{\left(\vec{\nabla}\left(u u^{*}\right)\right)^{2}}\right)+i \tilde{M}\left(\frac{\left(u u^{*}\right) \Delta\left(u u^{*}\right)}{\left(\vec{\nabla}\left(u u^{*}\right)\right)^{2}}\right)\right) u .
$$

Thus, equation (18) admits an invariance algebra which coincides with the invariance algebra of the linear Schrödinger equation with the arbitrary functions $M, N$. 
Note 1 With certain particular $M$ and $N$ the symmetry of system (18) can be essentially extended. E.g., if in (18) $N=\frac{1}{2}$, then the second equation of the system (equation for the phase) will be the Hamilton-Jacobi equation [5].

Let us consider some forms of the continuity equation (1) for equation (18).

Case 1. If $M=0$, then for solutions of equation (18) equation (1) holds true, where the density and current can be defined in the classical way (9).

Case 2. If $\Delta R M=-\lambda\left(\Delta R+\frac{(\vec{\nabla} R)^{2}}{R}\right)$, then for solutions of equation (18), the continuity equation (1), (6), (7) (or the Fokker-Planck equation (8), (9)) is valid.

Case 3. If $M$ is arbitrary then for solutions of equation (18), the continuity equation is valid, where the density and current can be defined by the conditions

$$
\rho=u u^{*}, \quad \vec{\nabla} \cdot \vec{j}=\frac{\partial}{\partial x_{k}}\left(-\frac{1}{2} i\left(\frac{\partial u}{\partial x_{k}} u^{*}-u \frac{\partial u^{*}}{\partial x_{k}}\right)\right)-2|u| \Delta|u| M\left(\frac{|u| \Delta|u|}{(\vec{\nabla}|u|)^{2}}\right) .
$$

Thus, we constructed wide classes of the nonlinear Schrödinger-type equations which is invariant with respect to algebra (13) (maximal invariance algebra of the linear Schrödinger equation) and for whose solutions the continuity equation (1) is valid.

The authors would like to thank the INTAS, SOROS, DKNT of Ukraina foundations for financial support. V. Boyko also would like to thank the AMS for financial support.

\section{References}

[1] Fushchych W.,Shtelen W. and Serov N. Symmetry Analysis and Exact Solutions of Equations of Nonlinear Mathematical Physics, Dordrecht, Kluwer Academic Publishers, 1993, 436 p.

[2] Doebner H.-D. and Goldin G.A., Properties of nonlinear Schrödinger equations associated with diffeomorphism group representations, J. Phys, A.: Math. Gen., 1994, V.27, 1771-1780.

[3] Fushchych W. I. and Cherniha R. M., Galilei-invariant nonlinear equations of the Schrödinger-type and their exact solutions I, II, Ukrainskyi Matem. Zhurn. (Ukrainian Math. J.), 1989, V.41, 1349$1357 ; 1687-1694$.

[4] Fushchych W.I. and Cherniha R.M., Galilei-invariant systems of nonlinear systems of evolution equations, J. Phys. A: Math.Gen., 1995, V.28, 5569-5579.

[5] Fushchych W., Cherniha R. and Chopyk V., On unique symmetry of two nonlinear generalizations of the Schrödinger equation, J. Nonlin. Math. Phys., 1996, V.3, N 3-4, 296-301.

[6] Fushchych W.I., Chopyk V., Nattermann P., Scherer W., Symmetries and reductions of nonlinear Schrödinger equations of Doebner-Goldin type, Reports on Math. Phys., 1995, V.35, N 1, 129-138.

[7] Vigier J.-P., Particular solutions of a nonlinear Schrödinger equation carrying particle-like singularities represent possible models of de Broglie's double theory, Phys. Lett. A, 1989, V.135, 99-105.

[8] Schuh D., Chung K.-M., Hartman H.N., Nonlinear Schrödinger-type field equation for the description of dissipative systems, J. Math. Phys., 1984, V.25, N 4, 786. 